\title{
Periodic variability of the mainline hydroxyl masers in G9.62+0.20E
}

\author{
S. Goedhart ${ }^{1,2 \star}$, R. van Rooyen ${ }^{1,2}$, D.J. van der Walt ${ }^{2}$, J.P. Maswanganye ${ }^{2}$, \\ A. Sanna ${ }^{3}$, G.C. MacLeod ${ }^{4,5}$ and S.P. van den Heever ${ }^{4}$ \\ ${ }^{1}$ South African Radio Astronomy Observatory, 2 Fir Street, Black River Park, Observatory, 7925, South Africa \\ ${ }^{2}$ Center for Space Research, North-West University, Potchefstroom campus, Private Bag X6001, Potchefstroom, 2520, South Africa \\ 3 Max-Planck-Institut für Radioastonomie, Auf dem Hügel 69, D-53121 Bonn, Germany \\ 4 Hartebeesthoek Radio Astronomy Observatory, PO Box 443, Krugersdorp, 1740, South Africa \\ ${ }^{5}$ The University of Western Ontario, 1151 Richmond Street. London, ON N6A 3K7, Canada
}

Accepted 2019 March 7. Received 2019 February 27; in original form 2018 December 20

\begin{abstract}
We present the results of a monitoring campaign using the KAT-7 and HartRAO 26m telescopes, of hydroxyl, methanol and water vapour masers associated with the highmass star forming region G9.62+0.20E. Periodic flaring of the main line hydroxyl masers were found, similar to that seen in the 6.7 and $12.2 \mathrm{GHz}$ methanol masers. The $1667 \mathrm{MHz}$ flares are characterized by a rapid decrease in flux density which is coincident with the start of the $12.2 \mathrm{GHz}$ methanol maser flare. The decrease in the $\mathrm{OH}$ maser flux density is followed by a slow increase till a maximum is reached after which the maser decays to its pre-flare level. A possible interpretation of the rapid decrease in the maser flux density is presented. Considering the projected separation between the periodic methanol and $\mathrm{OH}$ masers, we conclude that the periodic 12.2 methanol masing region is located about $1600 \mathrm{AU}$ deeper into the molecular envelope compared to the location of the periodic $\mathrm{OH}$ masers. A single water maser flare was also detected which seems not to be associated with the same event that gives rise to the periodic methanol and $\mathrm{OH}$ maser flares.
\end{abstract}

Key words: masers, star:formation, ISM:clouds, H II regions, radio lines:ISM

\section{INTRODUCTION}

To date there are at least 20 known periodic methanol masers (Goedhart et al. 2009; Araya et al. 2010; Szymczak et al. 2011; Goedhart et al. 2014; Fujisawa et al. 2014; Szymczak et al. 2015; Maswanganye et al. 2015; Szymczak et al. 2016; Maswanganye et al. 2016; Sugiyama et al. 2017) including one source which shows quasi-periodic variations in both methanol and formaldehyde (Araya et al. 2010). The formaldehyde and methanol variations were simultaneous and showed very close correspondence to each other, which the authors speculated was due to modulation of the infrared radiation field by periodic accretion in a binary system. Green et al. (2012) found a possible indication of periodic variability in the hydroxyl masers in G12.89+0.49, where the hydroxyl masers may have undergone a periodic drop in intensity. However, their results were not conclusive since the time series was undersampled. Szymczak et al. (2016) found anti-correlated variations in the water and methanol masers

^ E-mail: sharmila@ska.ac.za in G107.298+5.639 even though there are good spatial and velocity overlap between the two masers. Methanol and hydroxyl masers are thought to share a similar (radiative) pump mechanism (Cragg et al. 2002) and are often found in close spatial proximity, while water masers are collisionally pumped and require high density environments. Thus, correlated variability may be expected between hydroxyl and methanol masers. However, since the two molecules have different energy level structures, they may not necessarily respond in exactly the same way to changes in the pumping radiation field. The fact that several of the periodic methanol maser sources also have associated hydroxyl masers allows us to investigate to what extent the hydroxyl masers also show periodic flaring and therefore to possibly determine whether the flaring is due to changes in the maser amplification or in the background seed photon flux.

A number of known periodic methanol masers were monitored on a weekly basis at 1665 and $1667 \mathrm{MHz}$ as part of the seven-element Karoo Array Telescope (KAT7 ) science verification programme. This paper focuses on G9.62+0.20E, which showed clear evidence of variability 
in the hydroxyl masers during the first year of monitoring. It was subsequently monitored on a daily basis during the expected June 2014 and February 2015 methanol maser flares while simultanously monitoring the 6.7 and 12.2 $\mathrm{GHz}$ methanol and $22.2 \mathrm{GHz}$ water masers using the Hartebeesthoek Radio Astronomy Observatory (HartRAO) 26m telescope.

G9.62+0.20E is a high-mass star forming region with a hypercompact $\mathrm{H}$ II region harbouring at least one massive star in an early evolutionary phase (Garay et al. 1993). It has a number of masers projected against the HII region Class II methanol masers, water masers and hydroxyl masers (Sanna et al. 2015) and is located at a distance of $5.2 \mathrm{kpc}$ from the Sun (Sanna et al. 2009). It was the first methanol maser source discovered to show periodic variations (Goedhart et al. 2003), with a best-fit period of 243.3 days (Goedhart et al. 2014). Simultaneous flares in methanol have been observed at 6.7-, 12.2- and $107 \mathrm{GHz}$ (van der Walt et al. 2009). One explanation of the methanol maser flare profiles is by the variation of the free-free continuum flux in the background $\mathrm{H}$ II region due to variable ionizing radiation associated with shocked winds from a colliding wind binary system with a non-zero orbital eccentricity (van der Walt 2011). However, it has been argued by Parfenov \& Sobolev (2014) that the same effect can be produced by variations of the dust temperature in an accretion disk around a forming binary system, while Inayoshi et al. (2013) suggest that pulsational instabilities of massive protostars could arise during rapid accretion. Singh \& Deshpande (2012) investigated the possibility of bipolar outflows in young binary systems as a possible explanation for some of the periodic methanol masers. See van der Walt et al. (2016) for a further discussion.

The $\mathrm{OH}$ monitoring of $\mathrm{G} 9.62+0.20 \mathrm{E}$ revealed complex behaviour across multiple $\mathrm{OH}$ maser features, with some features showing a pronounced drop in power at the same time that the methanol masers started to flare. Not all of the hydroxyl or methanol maser features flare, and since the relative positions of all the maser species and the background H II region is known (Sanna et al. 2015), the regions undergoing periodic flares can be isolated. It was found that the flaring methanol and $\mathrm{OH}$ masers are, in projection, about 1600 AU apart. The decay of the flaring $\mathrm{OH}$ masers can described in terms of the variation of the free-free emission from a recombining hydrogen plasma, similar to what is found for the methanol masers in G9.62+0.20E.

\section{OBSERVATIONS AND DATA REDUCTION}

\section{$2.1 \quad$ KAT-7}

The seven-dish Karoo Array Telescope (Foley et al. 2016) was built as an engineering prototype for the 64-dish MeerKAT array in the Karoo region of the Northen Cape, South Africa. It consists of $712-\mathrm{m}$ diameter dishes with prime focus linearly-polarised receivers covering a frequency range of 1.2 to $1.95 \mathrm{GHz}$. It is a compact array, with a maximum baseline of $186 \mathrm{~m}$ and shortest baseline of $26 \mathrm{~m}$. The system temperature of the antennas is approximately $30 \mathrm{~K}$, and apperture efficiency is on average $65 \%$.

The $\mathrm{OH}$ maser monitoring observations started during the early stages of spectral line commissioning on KAT-7 in February 2013. We used the c16n2M4k correlator mode, which gives a velocity resolution of $68 \mathrm{~m} \mathrm{~s}^{-1}$ at the $\mathrm{OH}$ rest frequencies. Since May 2013, observations at 1665 and 1667 $\mathrm{MHz}$ were interleaved in a single schedule block using an LST-based scheduling mechanism, which ensures consistent uv-coverage from one observation to the next and ensures that observations at both frequencies are executed quasisimultaneously. Prior to this observations at each frequency were scheduled separately, preferably within the same day. The total integration time on source at each frequency was initially 20 minutes and subsequently increased to up to 50 minutes for the weekly monitoring programme and up to 80 minutes for the daily observations whenever possible. Daily observations during predicted flares were dynamically scheduled subject to telescope availability. Not all antennas were always available, and integration time was increased when possible to compensate. The typical rms noise achieved ranges from 0.15 to $0.2 \mathrm{Jy}$. The typical beam size is $\sim 3$ arcmin thus the masers are unresolved and the relative positions of the masers cannot be measured due to the poor resolution.

PKS 1934-638 - the flux and bandpass calibrator - was observed for five minutes every hour. PKS1730-130 was used as the gain calibrator and was observed once every ten minutes for one minute.

The data were calibrated following standard interferometric calibration procedures using CASA (Mcmullin et al. 2007). The bandpass response turned out to be stable in time so all scans were combined to produce a single bandpass calibration table. In order to avoid introducing noise from the bandpass calibration into the spectrum (the continuum emission is similar in strength to the bandpass calibrator) the bandpass solution was smoothed using a 3rd-order polynomial in both phase and amplitude. We were unable to obtain a reliable polarisation calibration in the narrow band mode due to insufficient signal to noise on the polarisation calibrator 3C286 so only the Stokes I product is considered here.

KAT-7 has a 1 square degree field of view and excellent sensitivity to low surface brightness emission. This results in a complex continuum image. Spectral line data cubes were imaged after continuum subtraction in the $\mathrm{u}-\mathrm{v}$ plane. The sheer volume of the data necessitated automated data reduction and imaging. The first 'quick-look' image cubes produced had imaging artefacts (spurious source detections in isolated channels) and higher rms noise than expected in some channels. To address this problem, a deep image was made of the field by combining all available observations at the time - some 60 hours of data on-source - in order to optimise the imaging parameters. There are five spectral line sources in the field of view, including a deep absorption feature, which were fully characterised to determine the appropriate velocity ranges for continuum subtraction, and to create a velocity-dependent CLEAN mask for non-interactive image deconvolution. The clean threshold was determined by measuring the rms noise in an emission-free channel. This approach led to much better and more consistent imaging quality even when uv-coverage was reduced.

Data quality was severely compromised by solar interference during December and early January when first the gain calibrator and then the target were less than 12 degrees 
from the Sun. We have discarded all data taken during this period.

\subsection{Single dish observations}

Concurrent monitoring of the methanol and water masers was done using the Hartebeesthoek Radio Astronomy HartRAO 26-m telescope, subject to scheduling constraints.

Flux calibration at 6.7 and $12.2 \mathrm{GHz}$ was done by daily drift scans on Virgo A and Hydra A using the flux scale of Ott et al. (1994). Amplitude corrections due to pointing errors were calculated by offset observations to the east-west and north-south half-power points. Bandpass calibration at 6.7 and $12.2 \mathrm{GHz}$ was done by frequency switching, while position-switching was done at $22 \mathrm{GHz}$ to accommodate a potentially wider velocity spread. The pointing offsets at 22 $\mathrm{GHz}$ were greater than half a beamwidth at times, making it impossible to derive reliable amplitude correction since we detect the source in only one or two of the offset pointing positions. These observations have been discarded from the timeseries. The $22 \mathrm{GHz}$ flux densities were also corrected for atmospheric absorption using water vapour radiometer and environmental data. Jupiter was used as a flux calibrator.

\section{RESULTS}

Figure 1 shows the spectra and range of variation of the $\mathrm{OH}$ masers at 1665 and $1667 \mathrm{MHz}$, the methanol masers at 6.7 and $12.2 \mathrm{GHz}$ and the water masers at $22 \mathrm{GHz}$. Variability is seen in the same velocity ranges for the methanol and hydroxyl transitions, between 1 to $3 \mathrm{~km} \mathrm{~s}^{-1}$. The water masers are highly variable but have a different and larger velocity range.

\subsection{Hydroxyl maser time-series}

Figure 2 shows the continuum image of the field with locations and identification of spectral line sources marked in red. Note that primary beam correction has not been applied to this image. At L-band we are detecting optically thin, evolved $\mathrm{H}$ II regions. Continuum emission is visible at the G9.62+0.20 site and is most likely dominated by components A and B (Garay et al. 1993), which are more evolved and extended $\mathrm{H}$ II regions in the complex.

Time-series were generated by fitting a 2-dimensional Gaussian to channels of interest in the image plane. The uncertainty in the measured amplitude was calculated by measuring the rms noise in an outer quadrant of the image for that channel. We generated time-series for all velocity channels which had amplitudes greater than three times the rms noise. Figures 3 and 4 show the time-series for the 1665 and $1667 \mathrm{MHz}$ data, respectively. The KAT-7 data suffers from heavy spectral blending, which could not be resolved by fitting individual Gaussian components to the spectra. Instead we plot the measured flux density in each channel. We have grouped channels by visual inspection of both the spectral structure and the characteristics of the variability, which appears markedly different as we move between spots in the general star forming region. The most notable behaviour is seen in the $1667 \mathrm{MHz}$ transition in the peak velocity channels at $\sim 1.7 \mathrm{~km} \mathrm{~s}^{-1}$. These features show a drop in intensity

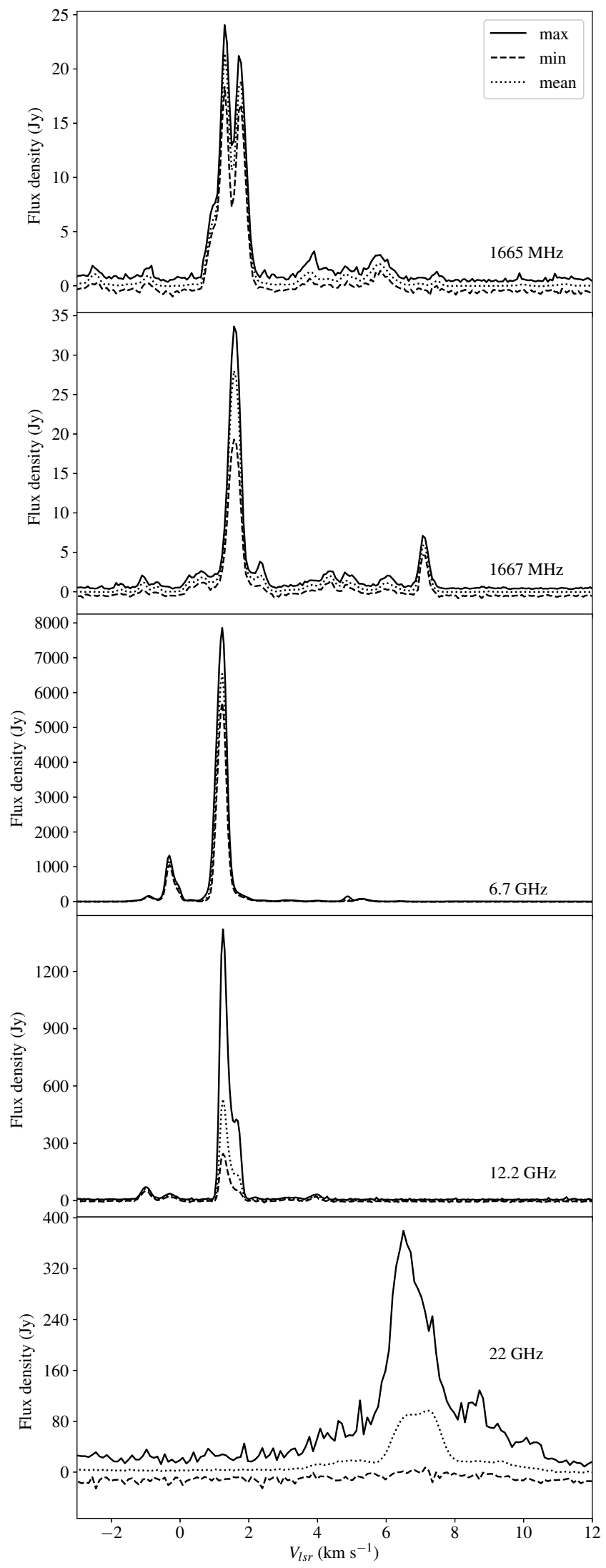

Figure 1. Spectra of the maser transitions monitored, showing the range of variation in each velocity channel. 


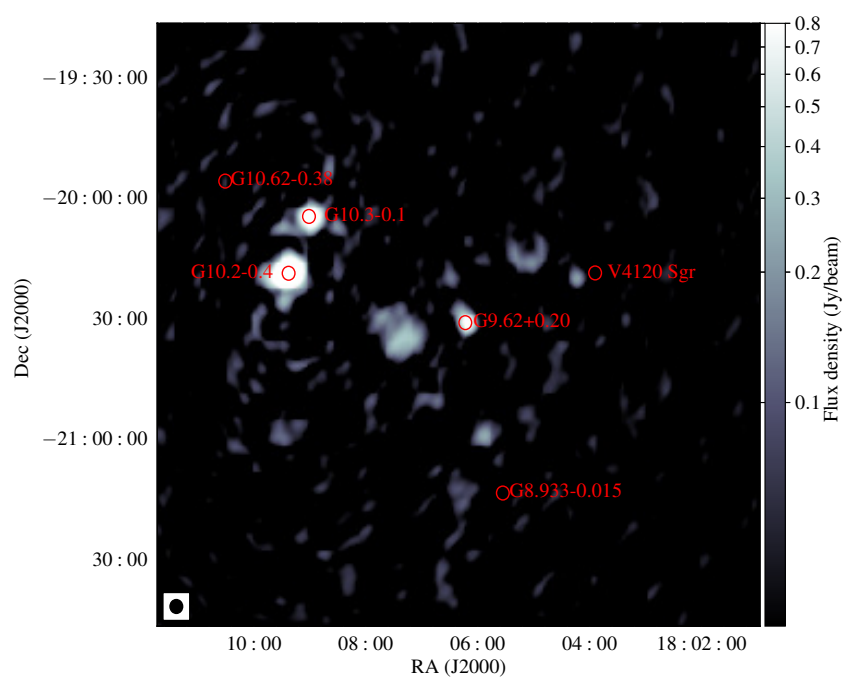

Figure 2. Overview of sources detected in the field. The colourscale shows the continuum image, Locations of spectral line sources are indicated by the red circles. The size of the circles indicates the synthesised beam size.

at the time that the methanol masers start to flare, followed by a sharp rise, and a gradual decay. In contrast, the features at $\sim 2 \mathrm{~km} \mathrm{~s}^{-1}$ show a flare profile that is more similar to that of the methanol masers.

\subsection{Comparison with VLBI spectra and radio continuum positions}

Since the individual hydroxyl maser spots cannot be resolved by KAT-7 and there are multiple spots with overlapping velocities, we inspected the VLBI spectra more closely to see if they could be correlated with the temporal and velocity behaviour seen in the KAT-7 data. The radio continuum source E appears to have two components - E1 is the stronger peak over which the methanol masers are centred, while E2 is one-fifth of the strength of $\mathrm{E} 1$ and is located $\sim 1000 \mathrm{AU}$ in projection to the north-east (see Sanna et al. 2015).

Figure 5 shows the maser spotmap from Sanna et al. (2015), with zoomed insets on the various OH maser clumps. The $\mathrm{OH}$ masers are not co-located with the methanol masers; instead they are distributed in several clumps around the region. The clump to the north-east is on the far side of component E2. Other clumps are distributed offset from the peak of E2 to the south-east, south and west. The water masers are located between E2 and E1. It is also known that not all of the methanol masers flare (Goedhart et al. 2005), so comparison of the maser positions and their time-dependent behaviour may help to narrow down the origin of the periodic behaviour.

Figures 6 and 7 show the VLBA spectra, with the KAT7 spectra taken at the closest epoch (16 July 2013) for comparison. These spectra are used to inform the allocation of spectral channels to mapped features. The following discussion will work through the enumerated panels in Figures 3 and 4. Panel ' $a$ ' covers velocities in the range 0.67 to 1.08 $\mathrm{km} \mathrm{s}^{-1}$ (the shoulder on the $1665 \mathrm{MHz}$ spectrum) and shows relatively constant flux densities throughout the monitoring period. These channels may be part of features 18/19 in the western group but the VLBI spectra show a single channel of $2.5 \mathrm{Jy}$ in this velocity range while the the KAT-7 spectra show far more flux. It is not completely clear where panel 'b' channels originate. It may be a blend of spatially separate features. The velocity range covered by panels ' $b$ ' and 'c' -1.15 to $1.36 \mathrm{~km} \mathrm{~s}^{-1}$ - is the same as that of features 17, 18 and 19 in the western group, however the VLBI spectra recover significantly lower flux. It may be that the weak indication of flaring behaviour in panel ' $c$ ' is spectral blending with features 12 and 13 shown in panel ' $\mathrm{d}$ ', and that the western group does not flare. The features in panel 'e' are unambiguously in the eastern group. Panel 'e' probably shows the blending of features 12/13 with features 18 and 19 in the west. In panel ' $\mathrm{f}$ ', note the slight rise in flux density at 1.9 to $2.04 \mathrm{~km} \mathrm{~s}^{-1}$ during May-June 2014 and a possible similar event February 2015, prior to the expected periodic flare. These features are in the same velocity range as 16 , 18,19 to the west, or 20 and 21 in the east. The feature in panel ' $\mathrm{g}$ ', 3.62 to $3.9 \mathrm{~km} \mathrm{~s}^{-1}$, appears to arise from a region $\approx 3$ arcsec to the south of the peak of the Hil region (Fish et al. 2005) and are not shown in Sanna et al. (2015). These features do not show any sort of correlated variability with the other masers.

Now to consider the $1667 \mathrm{MHz}$ transition. Here the spectrum is simpler and the variability much more pronounced. Panel ' $h$ ' does not show any significant variation. It is not entirely clear where this emission arises since the VLBI spectra at this velocity range, 1.16 to $1.38 \mathrm{~km} \mathrm{~s}^{-1}$, do not recover much flux. It could belong to features 4,5 or 10 , all of which are in the south-eastern group. Panel ' $\mathrm{i}$ ' in the range 1.44 to $1.78 \mathrm{~km} \mathrm{~s}^{-1}$, shows very well demarcated drops in intensity, which occur at the onset of the methanol flare, as we will show in the next section. After the 'dip', the masers increase in strength to a level higher than the pre-flare level then slowly recover over a period of several months. Features in this velocity range include $1,2,4,5,7,9$ and 10 . However, considering peak flux densities the contribution must be predominantly from features 1, 2, 7 and 9 which are in the east. Panel ' $\mathrm{j}$ ' does not show much variation. This velocity range, 1.85 to $2.05 \mathrm{~km} \mathrm{~s}^{-1}$, may be covered by features 7 and 9 but its not clear why they would not flare if all the other masers in this region are flaring. Panel ' $\mathrm{k}$ ' shows very pronounced flaring behaviour in the velocity range 2.12 to $2.4 \mathrm{~km} \mathrm{~s}^{-1}$ but it is unclear where these features are in the VLBI map - they could be part of $7 \& 9$ or $4 \& 5$ in the south-east. The features in panel 'l', as with panel 'g', arise from a region 3 arcsec to the south (Fish et al. 2005).

To summarise the most variable features appear to be in the eastern group, closer to $\mathrm{H}$ II region component $\mathrm{E} 2$ and it is likely that none of the other $\mathrm{OH}$ maser groups are flaring.

\subsection{Comparison of $\mathrm{OH}$ and methanol maser flare profiles}

As already noted above, the $\mathrm{OH}$ maser flares have a "characteristic" dip in the flux density before the flare. Such behaviour has not been reported for the methanol maser flares. In Figure 8 a more detailed comparison between the $\mathrm{OH}$ and methanol maser flares is shown. The dip is seen to be significantly more pronounced for the $1667 \mathrm{MHz}$ maser than for the $1665 \mathrm{MHz}$ maser. It is rather interesting to note that 


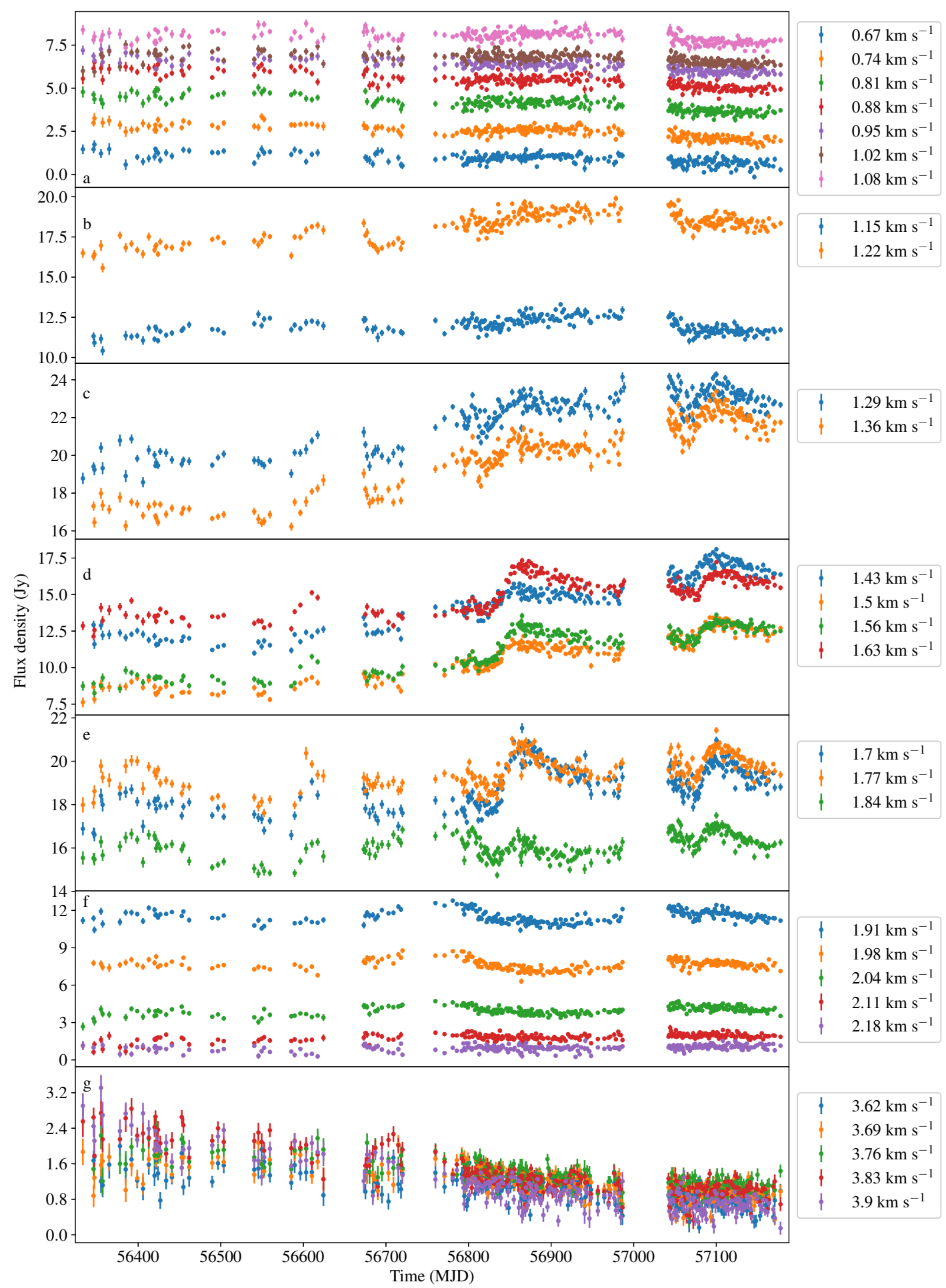

Figure 3. Time-series of individual velocity channels for the $1665 \mathrm{MHz} \mathrm{OH}$ masers. Plots have been split across channels to aid in visual identification of correlated behaviour.

for the $1667 \mathrm{MHz}$ maser the onset of the dip is very near to the start of the $12.2 \mathrm{GHz}$ maser flare. After the dip, the $\mathrm{OH}$ masers increase to a maximum after which they decay to the same level as before the onset of the $12.2 \mathrm{GHz}$ maser flare. The time intervals between the peak of the $12.2 \mathrm{GHz}$ maser and when the 1665 and $1667 \mathrm{MHz}$ masers reach their maxima are about 23 and 13 days respectively.
Given that after reaching a maximum, the $\mathrm{OH}$ masers recover to the same pre-flare level, a behaviour also seen for the methanol masers, we also tried to fit the decay part of the $\mathrm{OH}$ masers with equation A7 of van der Walt et al. (2009) as well as to the decay of the $12.2 \mathrm{GHz}$ methanol maser flare shown in Figure 8. The fits are shown as the blue solid lines in Figure 8. It is seen that for all three masers the decay 


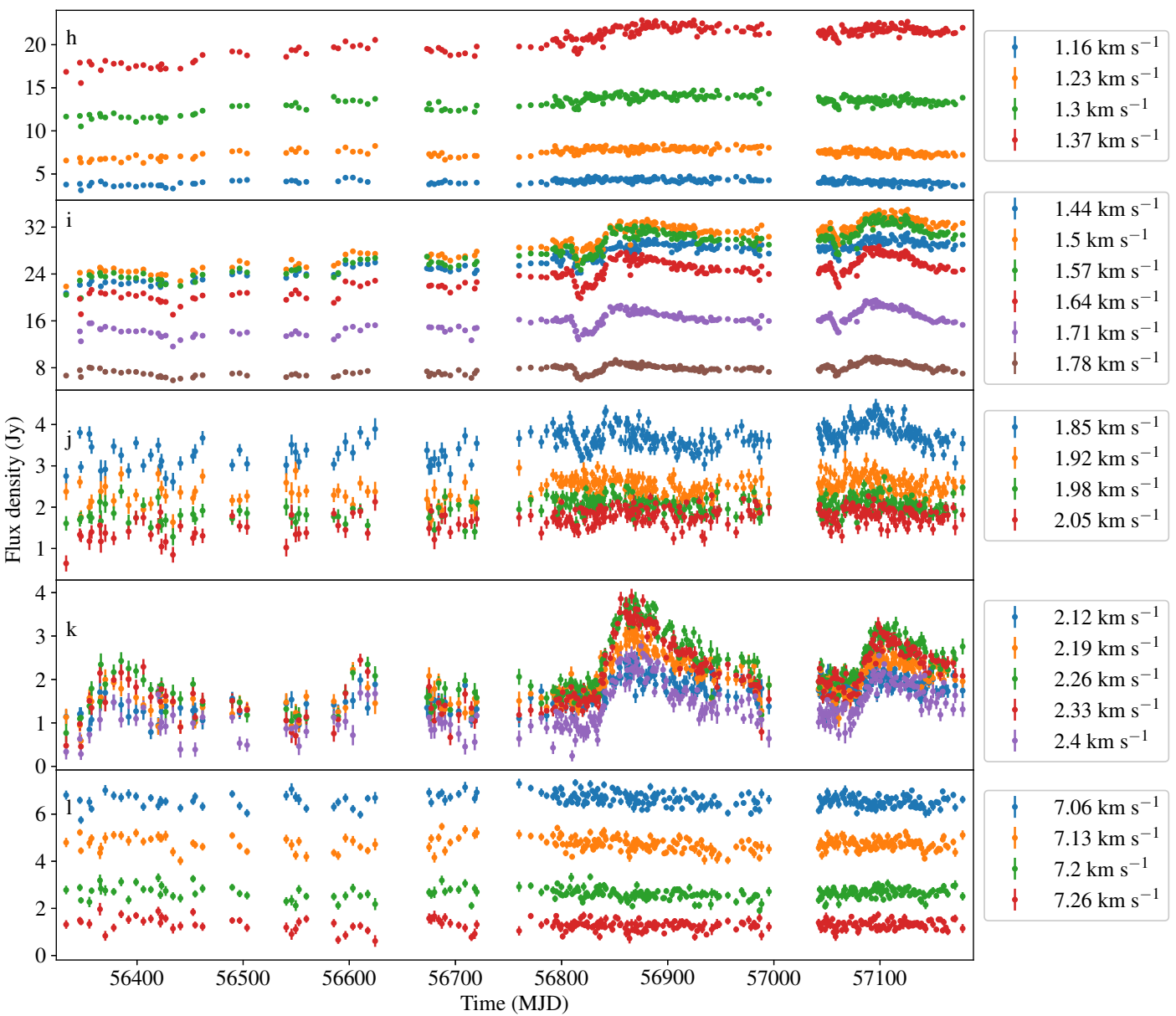

Figure 4. Time-series of individual velocity channels for the $1667 \mathrm{MHz} \mathrm{OH}$ masers. Plots have been split across channels to aid in visual identification of correlated behaviour.

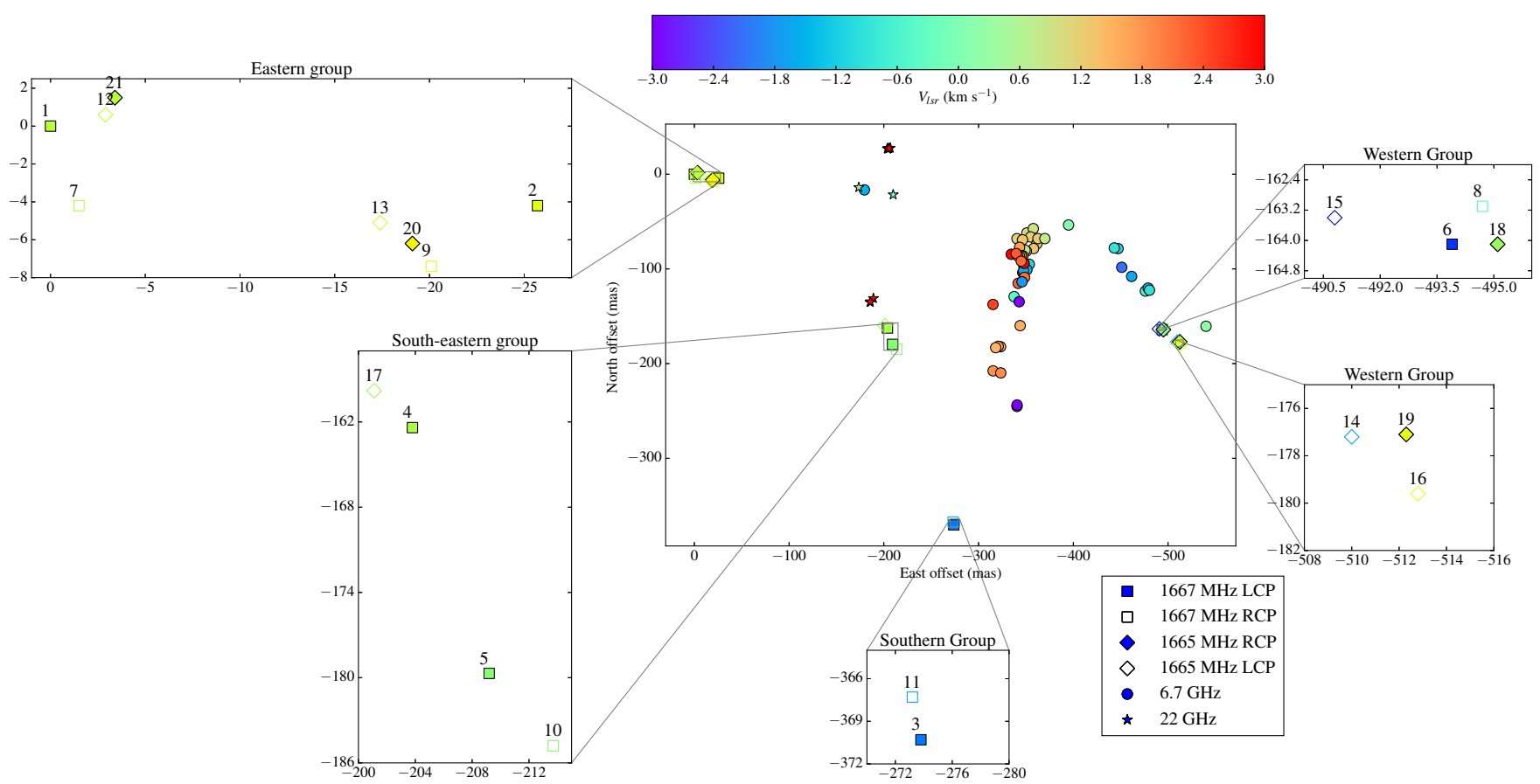

Figure 5. Maser spot positions as reported in Table 2 of Sanna et al. (2015). 
of the masers are described very well by eq. A7 of van der Walt et al. (2009). Assuming that the decay of the masers are indeed related to the recombination of a partially ionized hydrogen plasma, the fit allows us to obtain estimates of the quiescent state electron density $\left(n_{e, \star}\right)$ as well as the ratio $\left(u_{0}\right)$ of the electron density from where the recombination started and $n_{e, \star}$. The values of these two quantities obtained from the fits are shown in the respective panels of Figure 8 . The derived quiescent state densities range from $5.7 \times 10^{5} \mathrm{~cm}^{-3}$ for the $12.2 \mathrm{GHz}$ maser at $1.25 \mathrm{~km} \mathrm{~s}^{-1}$ to $1.54 \times 10^{5} \mathrm{~cm}^{-3}$ for the $1665 \mathrm{MHz} \mathrm{OH}$ maser. The order of magnitude of these densities are what is expected for very young $\mathrm{H}$ II regions. It is also seen that for the $\mathrm{OH}$ masers the value of $u_{0}$ as found from the fit is only 1.07, in agreement with the small amplitude of the flares. For comparison, $u_{0}=1.85$ for the strong $12.2 \mathrm{GHz}$ flare.

\subsection{Water maser monitoring}

Figure 9 shows a dynamic spectrum of the water maser observations. While the masers are variable, there does not appear to be any particular correlation with the methanol or hydroxyl, but the time-coverage during the September 2013 flare is extremely sparse due to bad telescope pointing. There are some transient features at $\sim 5 \mathrm{~km} \mathrm{~s}^{-1}$ and $\sim 8$ $\mathrm{km} \mathrm{s}^{-1}$ in the second flare cycle, which also appear to have slight changes in peak velocities very typical of water masers in an outflow.

In Figure 10 we show a more detailed comparison between the time series of the water- and $12.2 \mathrm{GHz}$ methanol masers between MJD 56700 and 57400. It is seen that the duration of the water maser flare covers a time interval approximately equal to the period of the $12.2 \mathrm{GHz}$ flares. The water and methanol maser flares do not seem to be related in the same way as seems to be the case for the $\mathrm{OH}$ and methanol maser flares.

\section{DISCUSSION}

There are quite a number of interesting aspects to note in G9.62+0.20. We first note, using Figure 1 of Sanna et al. (2015), that, in projection, the periodic methanol and $\mathrm{OH}$ masers are located approximately 330 and $1930 \mathrm{AU}$ from the continuum peak of the VLA A-array $7 \mathrm{~mm}$ emission. Although there are $\mathrm{OH}$ masers located closer to the continuum peak, these do not appear to be periodic at all. The question then is why the periodic $\mathrm{OH}$ masers are located so much further than the periodic methanol masers from the peak of continuum. As shown above, equation A7 of van der Walt et al. (2009) provides a very good fit to the decay of the 12.2 $\mathrm{GHz}$ methanol maser flare. The implication is that the variation in the electron density occurs at such a position that the $\mathrm{H}$ II region is optically thin for outward propagating 12.2 $\mathrm{GHz}$ photons in the direction of the masing gas. Since the optical depth is proportional to $\lambda^{2.1}$, it follows that at the projected position of the periodic methanol masers, the optical depth at $1665 \mathrm{MHz}$ is about 65 times greater than at $12.2 \mathrm{GHz}$. If, for example, the optical depth at $12.2 \mathrm{GHz}$ is 0.1 , it means that it is 6.5 at $1665 \mathrm{MHz}$ and therefore that all variability will be damped due to absorption in the $\mathrm{H}$ II region. Qualitatively, it is therefore expected that if there are periodic $\mathrm{OH}$ masers, that these will be located further from the core of the $\mathrm{H}$ II region compared to the periodic methanol masers. This might explain the relative projected positions of the periodic methanol and $\mathrm{OH}$ masers in G9.62+0.20E.

Perhaps the most intruiging aspect of the periodic $\mathrm{OH}$ masers in G9.62+0.20E is the pronounced sharp decrease (dip) in the maser flux density before the "flare". That the dip is a real feature is clear from the fact that it is seen in two flares and is also present in the $1665 \mathrm{MHz}$ masers. As noted above, the periodic methanol masers do not show this behaviour. If, as proposed by van der Walt et al. (2009), van der Walt (2011) and, van der Walt et al. (2016), the periodicity of the methanol masers in G9.62+0.20E are driven by a colliding-wind binary system and that the flares are due to changes in the background free-free emission from the $\mathrm{H}$ II region, it is required that the dip should also be explained within the same framework. We consider two possibilities.

(a) The periodicity is due to a CWB associated with component E1: Although no quantitative explanation can be given, qualitatively we note the following. First, from the maps of Sanna et al. (2015) it is seen that the periodic $\mathrm{OH}$ masers are projected significantly ( 1600 AU) further from the core of the Hil region E1 than the periodic methanol masers. Should both masers amplify the free-free emission from the background $\mathrm{H}$ II region, it follows that they probe two completely different parts of the $\mathrm{H}$ II region. The ionization structure of an UCH II region as calculated with the photo-ionization code Cloudy (see eg. van der Walt 2011) shows that partially ionized gas extends beyond the ionization front. Within the framework of the CWB scenario, the lower energy ionizing photons from the hot shocked gas are absorbed at the ionization front and gives rise to the flaring of the methanol masers. Higher energy $(>100 \mathrm{eV})$ photons, on the other hand, can propagate beyond the ionization front due to the significantly lower photo-ionization cross section for HI. Ionization of hydrogen by these photons will result in higher average electron temperatures. In the optically thin case the free-free emission is proportional to the volume emissivity which, in turn, is proportional to $T_{e}^{-0.5}$. Raising the electron temperature of the plasma thus lowers the volume emissivity.

A possible explanation then for the dip in the $1667 \mathrm{MHz}$ maser flux density is that, because of the higher energies of the ionizing photons, the electron temperature is raised significantly during the ionization event in the gas against which the $\mathrm{OH}$ maser is projected, resulting in a decrease in the free-free emission. After the pulse of ionizing photons has passed, the electrons cool to the equilibrium temperature giving rise to an increase in the free-free emission and therefore also in the maser emission. Simultaneous to the increase in the electron temperature, the electron density also increases due to the pulse of ionizing photons. The exact profile of the flare (which includes the dip) depends on the magnitude of the change in electron temperature, the cooling rate of the electrons and the ionization rate associated with the pulse of ionizing photons. The observed peak of the $\mathrm{OH}$ maser flares therefore does not necessarily indicate the time of the peak of the pulse of ionizing photons as in the case of the methanol maser flares. The difference in time between the peaks of the methanol and $\mathrm{OH}$ maser flares, might therefore not be due to a geometric delay only (see below) but also to other physical effects. The required change in 
electron temperature to explain the magnitude of the dip can be estimated by considering the ratio of flux densities of the $1667 \mathrm{MHz}$ maser before the dip and at the minimum of the dip (Figure 8). It then follows that the electron temperature has to be raised by a factor of 1.8 to explain the observed decrease in the maser flux density. Thus, for example, if before the dip the electron temperature was $5000 \mathrm{~K}$, it has to be raised to $9000 \mathrm{~K}$ in order to explain the decrease seen in the maser flux density.

(b) The periodicity is associated with component E2. Given that the decay of all three masers are described very well in terms of the recombination of a hydrogen plasma, it is reasonable to assume that component E2 must then also be a periodic source of ionizing photons which influence the flux of seed photons for both the methanol and $\mathrm{OH}$ masers. Since the methanol masers are projected against E1, it is therefore required that the ionizing photons propagate from E2 to the position against which the periodic methanol masers are projected. However, it is clear from Sanna et al. (2015) that there is very little ionized gas associated with E2, which means that a significant fraction of the flux of the periodically produced ionizing photons will be absorbed close to E2. It is therefore difficult to see how a source of periodically varying ionizing photons located at E2 will influence that part of E1 against which the periodic methanol masers are projected.

Having excluded E2 as the driving source for the periodic methanol and $\mathrm{OH}$ masers, we finally note the following. From Figure 8 it is seen that although the peak of the $1667 \mathrm{MHz}$ flare lags that of the $12.2 \mathrm{GHz}$ flare by about 13 days, the dip of the $1667 \mathrm{MHz}$ maser occurs almost simultaneously with the onset of the $12.2 \mathrm{GHz}$ methanol maser flare. In view of the above possible explanation for the dip, it seems reasonable to regard the onset of the $12.2 \mathrm{GHz}$ flare and the almost simultaneous sharp decrease of the 1667 $\mathrm{MHz}$ maser flux density to be caused by the same ionization event. However, due to the difference in projected distances from the core of the H II region, we then expect the dip of the $\mathrm{OH}$ maser flares to be delayed by about nine days with respect to the onset of the $12.2 \mathrm{GHz}$ flare. Considering the very simple geometry in Figure 11, the time difference between the methanol and hydroxyl flares is given by $\Delta t=\left(r_{\mathrm{B}}-r_{\mathrm{A}}+z_{\mathrm{OH}}-z_{\mathrm{CH}_{3} \mathrm{OH}}\right) / c$. For the present case $\Delta t \sim 0$, from which follows that $z_{\mathrm{CH}_{3} \mathrm{OH}}-z_{\mathrm{OH}} \approx r_{\mathrm{B}}-r_{\mathrm{A}}$. Our observations of the methanol and $\mathrm{OH}$ maser flares therefore suggest that the masing region of the periodic $12.2 \mathrm{GHz}$ methanol masers are located about 1600 AU further from the "surface" of the H II region into the molecular envelope compared to the location of the periodic $\mathrm{OH}$ masers.

As noted above, a water maser flare occured that overlapped in time with a $12.2 \mathrm{GHz}$ methanol maser flare. It seems as if a comparison between the water and methanol maser flares similar to that between $\mathrm{OH}$ and methanol masers cannot be made. Although the water maser flare overlaps in time with the $12.2 \mathrm{GHz}$ methanol maser, the water maser flare clearly starts before the methanol maser flare. Also, the decay time of the water maser flare is significantly different from that of the $\mathrm{OH}$ and methanol maser flares and does not suggest any causal relation with the methanol and $\mathrm{OH}$ flares.

To our knowledge, the results presented above are the first conclusive observational evidence of periodic variabil- ity of $\mathrm{OH}$ masers associated with a high-mass star forming region. Green et al. (2012) found an indication of periodicity in the $\mathrm{OH}$ masers toward G12.889+0.489 (which has a 29.5 day period in methanol), but the time-series were too undersampled to obtain detailed cycle profiles and no contemporaneous methanol monitoring was done. It is interesting that the hydroxyl masers appear to undergo a drop in emission coincident with the expected minima of $6.7 \mathrm{GHz}$ methanol masers but a key difference is that the hydroxyl masers do not show any flares. G12.889+0.489 also differs from G9.62+0.20E in that it has somewhat irregular flaring behaviour in the methanol, but shows a well-defined minimum, which seems to be periodic (Goedhart et al. 2009), while the peaks of the methanol flares can occur any time in an 11-day window. It is quite likely, given the short period and the difference in the methanol maser light curves, that a different mechanism is modulating the maser intensity. It would undoubtedly be of great benefit to our understanding of these phenomena to conduct intensive monitoring of both maser species through one 29.5 day cycle in this source.

\section{SUMMARY AND CONCLUSIONS}

We presented the first conclusive observational evidence for the periodic variability of $\mathrm{OH}$ mainline masers associated with a high-mass star forming region. The $1667 \mathrm{MHz}$ masers show a pronounced dip in flux density which closely coincide with the onset of the $12.2 \mathrm{GHz}$ methanol maser flare. The decay of the $\mathrm{OH}$ maser flares, similar to that of the methanol maser flares, can be described very well by the decrease in the free-free emission from the background H II region as expected from a recombining hydrogen plasma. A possible explanation, within the framework of the colliding-wind binary scenario, for the dip in flux density of the $1667 \mathrm{MHz}$ masers is that it is due to an increase in electron temperature following the ionization of the outer regions of the $\mathrm{HII}$ region by photons with energy greater than about $100 \mathrm{eV}$. We also argued, within this framework that the masing region for the periodic $12.2 \mathrm{GHz}$ methanol masers are located about 1600 AU further from the "surface" of the H II region into the molecular envelope compared to the location of the periodic $\mathrm{OH}$ masers.

\section{REFERENCES}

Araya E., Hofner P., Goss W. M., Kurtz S., Richards a. M. S., Linz H., Olmi L., Sewiło M., 2010, ApJ, 717, L133

Cragg D. M., Sobolev A. M., Godrey P. D., 2002, MNRAS, 331, 521

Fish V. L., Reid M. J., Argon A. L., Zheng X., 2005, ApJS, 160, 220

Foley A. R., et al., 2016, MNRAS, 460, 1664

Fujisawa K., et al., 2014, PASJ, 66, 78

Garay G., Rodríguez L. F., Moran J. M., Churchwell E., 1993, ApJ, 418, 368

Goedhart S., Gaylard M. J., van der Walt D. J., 2003, MNRAS, $36, \mathrm{~L} 33$

Goedhart S., Minier V., Gaylard M. J., van der Walt D. J., 2005, MNRAS, 356, 839

Goedhart S., Langa M. C., Gaylard M. J., van der Walt D. J., 2009, MNRAS, 398, 995 
Goedhart S., Maswanganye J. P., Gaylard M. J., van der Walt D. J., 2014, MNRAS, 437, 1808

Green J. A., Caswell J. L., Voronkov M. A., McClure- Griffiths N. M., 2012, MNRAS, 425, 1504

Inayoshi K., Sugiyama K., Hosokawa T., Motogi K., Tanaka K. E. I., 2013, ApJ, 769, L20

Maswanganye J. P., Gaylard M. J., Goedhart S., van der Walt D. J., Booth R. S., 2015, MNRAS, 446, 2730

Maswanganye J. P., van der Walt D. J., Goedhart S., Gaylard M. J., 2016, MNRAS, 456, 4335

Mcmullin J. P., Waters B., Schiebel D., Young W., Golap K., 2007, Astronomical Data Analysis Software and Systems XVI, 376,127

Ott M., Witzel A., Quirrenbach A., Krichbaum T., Standke K., Schalinski C., Hummel C., 1994, A\&A, 284, 331

Parfenov S. Y., Sobolev A. M., 2014, MNRAS, 444, 620

Sanna A., Reid M. J., Moscadelli L., Dame T. M., Menten K. M., Brunthaler A., Zheng X. W., Xu Y., 2009, ApJ, 706, 464

Sanna A., et al., 2015, ApJ, 804, L2

Singh N. K., Deshpande A. A., 2012, in Booth R. S., Vlemmings W. H. T., Humphreys E. M. L., eds, IAU Symposium Vol. 287, Cosmic Masers - from OH to H0. pp 93-97

Sugiyama K., et al., 2017, PASJ, 69, 59

Szymczak M., Wolak P., Bartkiewicz a., van Langevelde H. J., 2011, A\&A, 531, L3

Szymczak M., Wolak P., Bartkiewicz a., 2015, MNRAS, 448, 2284

Szymczak M., Olech M., Wolak P., Bartkiewicz A., Gawroński M., 2016, MNRAS, 459, L56

van der Walt D. J., 2011, AJ, 141, 152

van der Walt D. J., Goedhart S., Gaylard M. J., 2009, MNRAS, 398, 961

van der Walt D. J., Maswanganye J. P., Etoka S., Goedhart S., van den Heever S. P., 2016, A\&A, 588, A47

This paper has been typeset from a $\mathrm{T}_{\mathrm{E}} \mathrm{X} / \mathrm{LAT}_{\mathrm{E}} \mathrm{X}$ file prepared by the author.

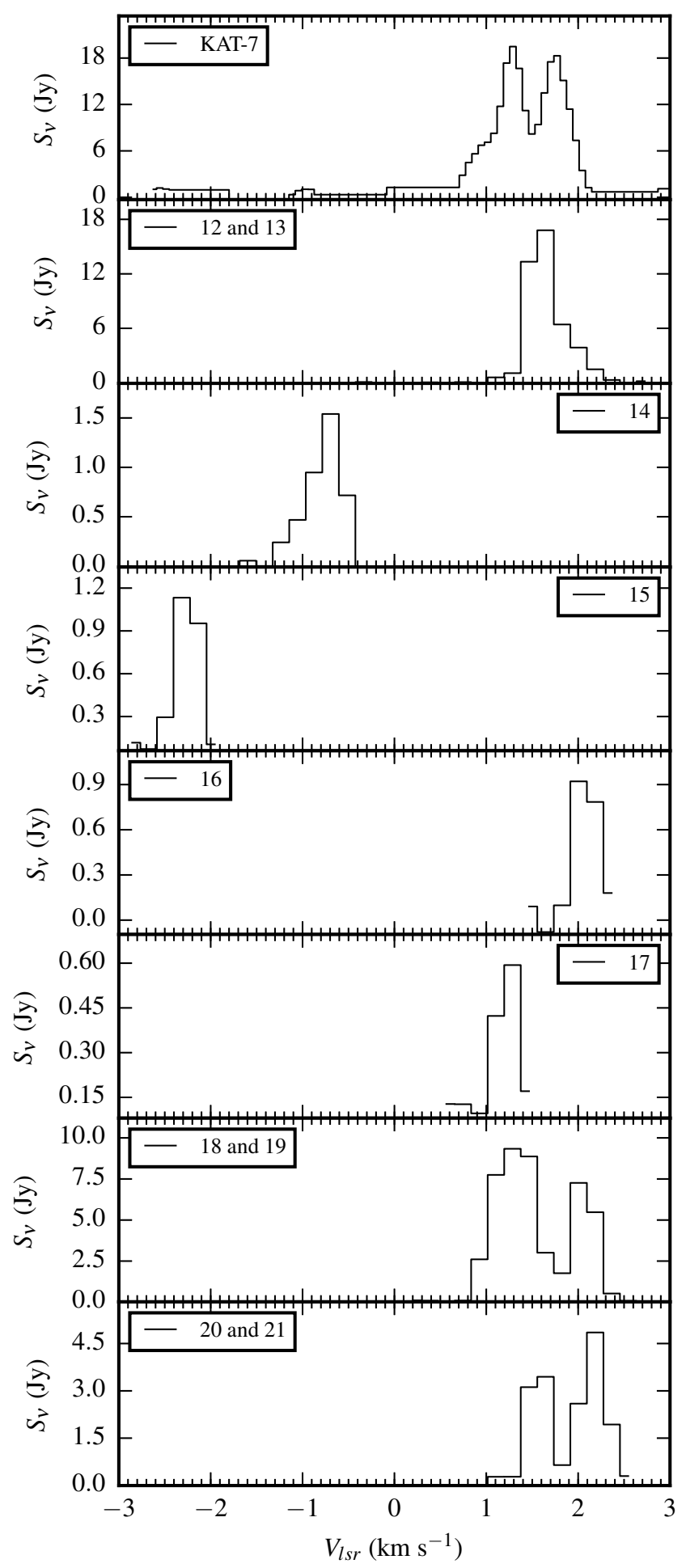

Figure 6. Spectra of individual $1665 \mathrm{MHz}$ maser features from Sanna et al. (2015). The top panel shows the KAT-7 spectrum from the nearest epoch (2013-07-16) 14 days later. 


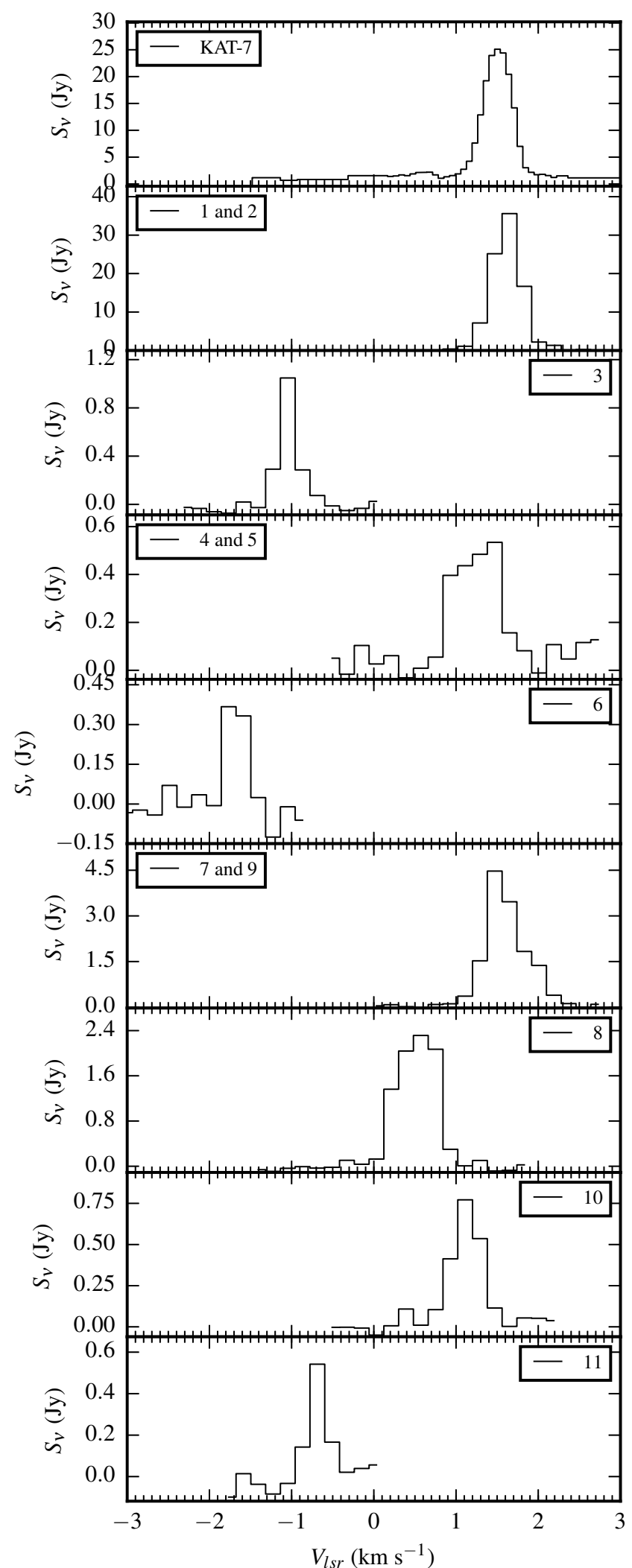

Figure 7. Spectra of individual $1667 \mathrm{MHz}$ maser features from Sanna et al. (2015). The top panel shows the KAT-7 spectrum from the nearest epoch (2013-07-16) 14 days later.

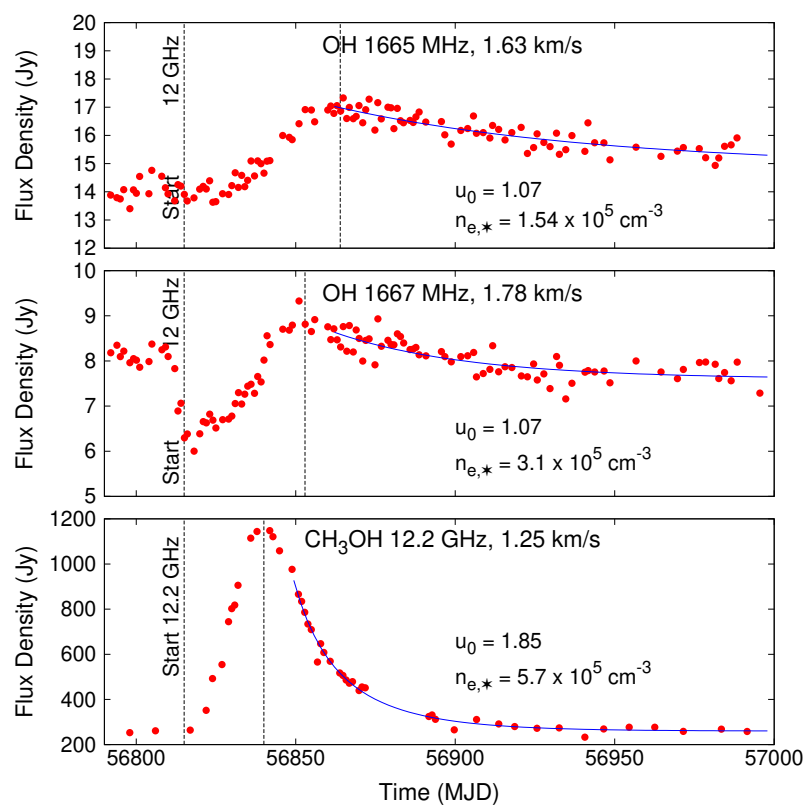

Figure 8. More detailed comparison of the profiles of the $\mathrm{OH}$ and methanol maser flares. The solid blue lines are the fits of eq. A7 of van der Walt et al. (2009) to the decay of the flares. See van der Walt et al. (2009) for the interpretation of $u_{0}$ and $n_{\boldsymbol{e}} \star \star$. 


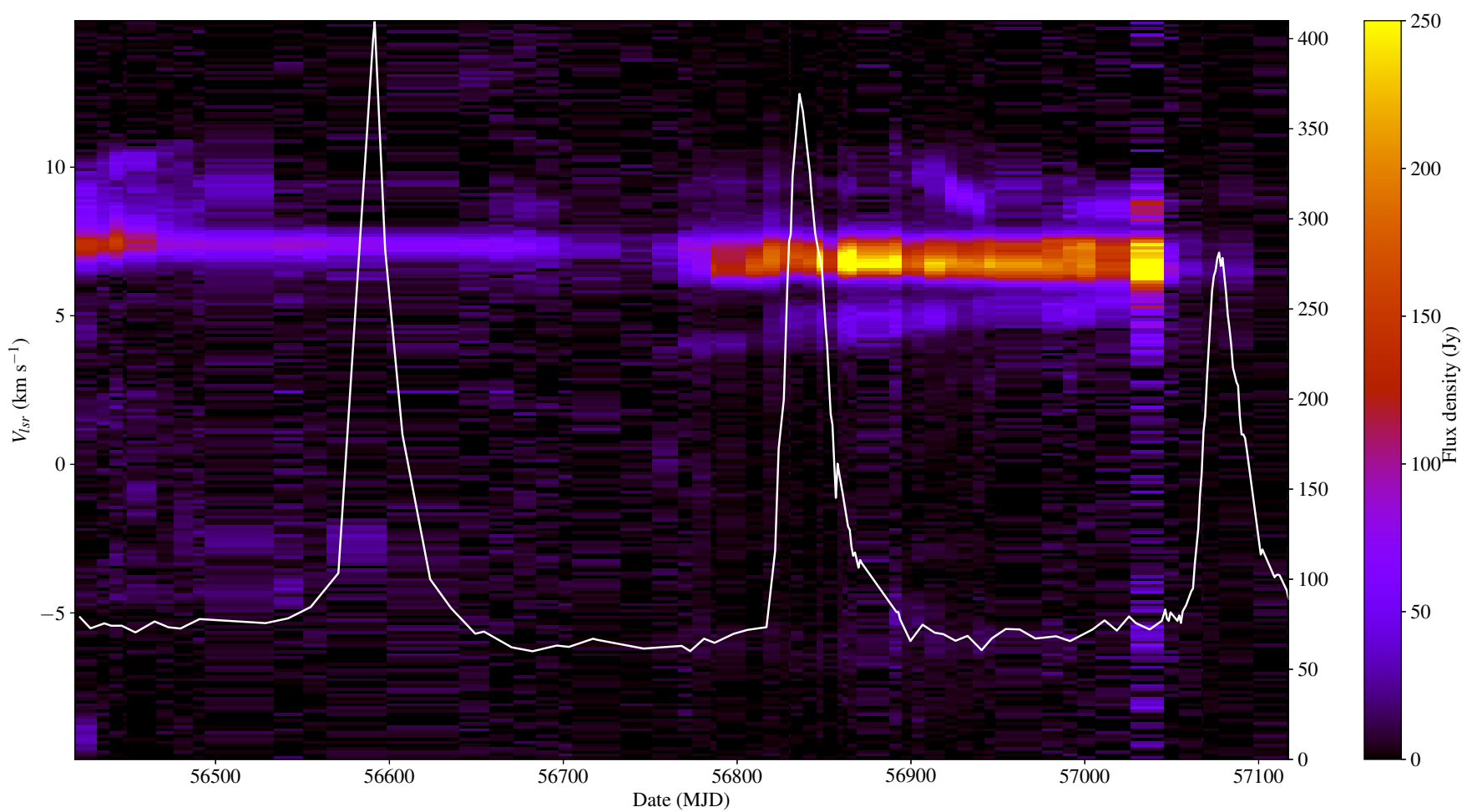

Figure 9. Flux density of water masers as a function of time and velocity in the colorscale plot. The white curve is the $12.2 \mathrm{GHz}$ methanol for comparison.

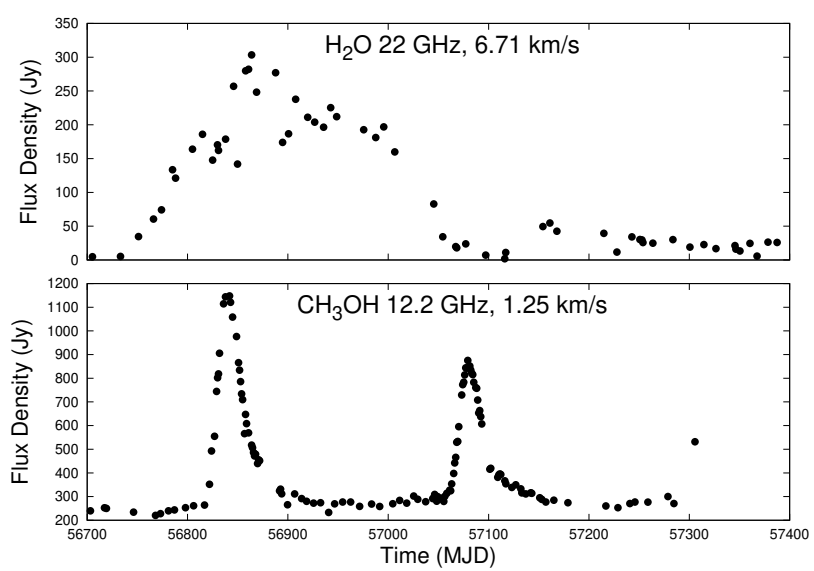

Figure 10. Comparison of water and $12.2 \mathrm{GHz}$ methanol maser flares

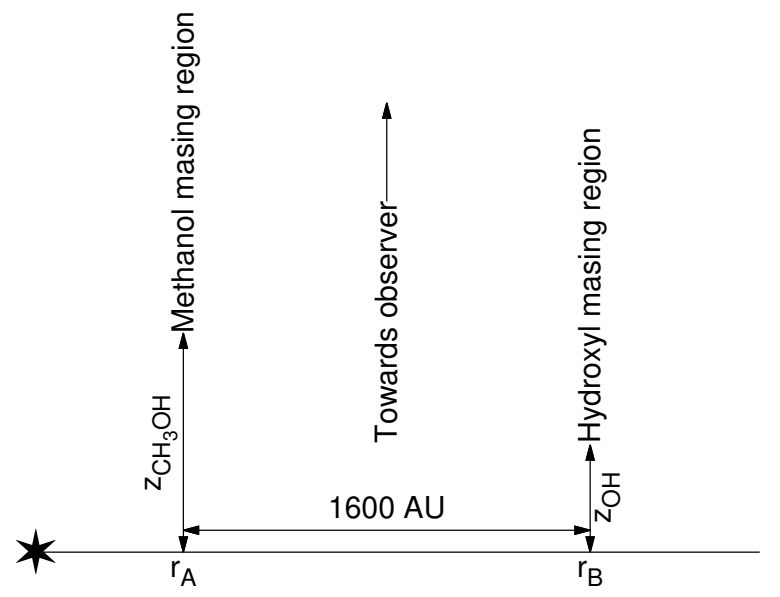

Figure 11. Simplified geometry for the locations of the periodic methanol and hydroxyl masers. The ionizing star is indicated by the star symbol. The "surface" of the H II region is assumed to be flat. The methanol and hydroxyl masers respectively respond to changes in the free-free emission from points A and B 